FACTA UNIVERSITATIS

Series: Mechanical Engineering Vol. 14, N 3 3, 2016, pp. 293 - 300

DOI: $10.22190 /$ FUME1603293K

Original scientific paper

\title{
THE BOUNDARY ELEMENT METHOD FOR VISCOELASTIC MATERIAL APPLIED TO THE OBLIQUE IMPACT OF SPHERES
}

\author{
UDC 539.3
}

\author{
Stephan Kusche \\ Department of System Dynamics and the Physics of Friction, TU Berlin, Germany
}

\begin{abstract}
The Boundary Element Method (BEM) for elastic materials is extended to deal with viscoelastic media. This is obtained by making use of a similar form of the fundamental solution for both the materials. Some considerations are attributed to the difference of the normal and the tangential contact problem. Both normal and tangential problems are furthermore assumed to be decoupled. Then the oblique impact of hard spheres with an incompressible viscoelastic half-space (linear standard-model) is studied. By assuming stick conditions during impact, one obtains the dependence of the two coefficients of restitution as functions of two input parameters. This result is expressed in an elegant and compact form of the fitting function.
\end{abstract}

Key Words: Contact Mechanics, Boundary Element Method, Linear Viscoelastic Material, Rebound Test, Oblique Impact, Tangential Problem

\section{INTRODUCTION}

The history of analytical research of the spheres' field of the impact started with Hertz [1] in 1882. His theory of normal contacts has been extended to tangential contact theories, considering partial slip [2-9]. Like in other fields of contact mechanics, a solution in the case of viscoelastic time dependent material behavior is difficult and its closed form has not been found yet. For the pure normal impact of spheres Hunter [10], Sabin [11] and Calvit [12] discovered some analytical solutions. These solutions are given in an implicit form; some kind of numerical treatment is needed in order to obtain results.

In the field of elastic contact mechanics, the Boundary Element Method (BEM) is well established. This method is based on the fundamental solution for elastic half-space. Since the fundamental solution for the viscoelastic half-space is known as well, it should be possible to adapt many procedures from elastic to viscoelastic problems. This can be achieved by using

Received September 22, 2016 / Accepted November 18, 2016

Corresponding author: Stephan Kusche

Institute of Mechanics, Berlin Institute of Technology, Str. d. 17. Juni 135, 10623 Berlin, Germany

E-mail: s.kusche@tu-berlin.de 
the Prony series for modeling the material behavior. In this paper the given approach is used to study the viscoelastic impact problem of spheres.

\section{PROBLEM DESCRIPTION}

The problem under consideration is the following: A sphere of mass $m$ and radius $R$ has initial velocities $v_{x 0}{ }^{s}, v_{z 0}{ }^{s}$ and an initial angular velocity $\omega_{0}{ }^{\mathrm{s}}$. After rebound from viscoelastic ground the velocities change to $v_{x 1}{ }^{s}, v_{z 1}{ }^{s}$ and $\omega_{1}{ }^{\text {s }}$ (see Fig. 1). These velocities have to be calculated.

To simplify this problem approach it will be assumed, that the normal- and the tangential-contact problems are decoupled. This assumption becomes true under two circumstances. Firstly, the sphere is a rigid body and the viscoelastic half-space is incompressible. Then the normal load will not lead to any tangential displacement and vice versa. Secondly, the displacement of the centre of the sphere in tangential direction $u_{x}{ }^{s}$ is assumed to be much smaller than radius $R$ of the sphere. Then only tangential contact force $F_{x}$ has to be taken into account for the calculation of the resulting moment acting on the sphere. Furthermore, the contact area will conserve a rotational symmetric shape and the action line of resulting normal force $F_{z}$ points toward the centre of the sphere.

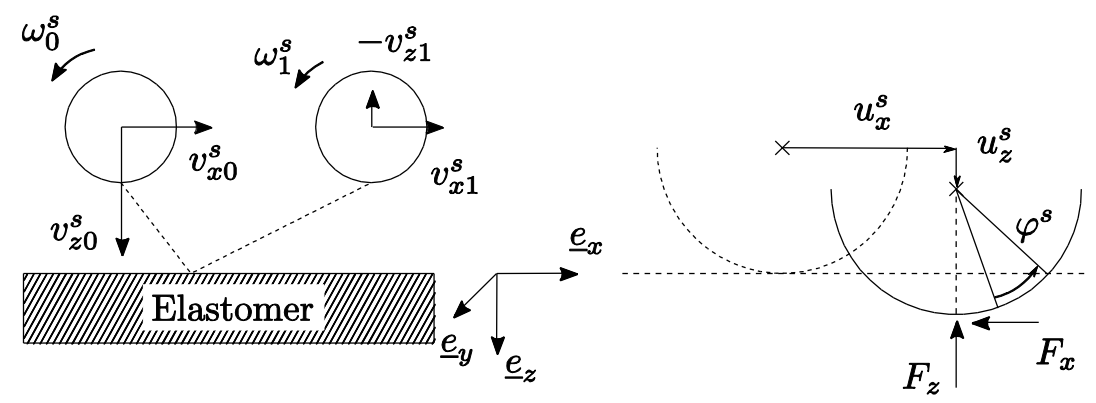

Fig. 1 Velocities before and after impact (left hand side). Displacement of the center of the sphere and resulting contact forces during impact (right hand side)

With these considerations tangential velocity $v_{x}^{s}$ and angular velocity $\omega^{\mathrm{s}}$ are not independent of each other. By using the principle of impulse and angular momentum one obtains:

$$
m \ddot{u}_{x}^{s}=-F_{x}, \quad \frac{2}{5} m R^{2} \ddot{\varphi}^{s}=-F_{x} R,
$$

and this results in:

$$
v_{x}^{s}-v_{x 0}^{s}=\frac{2}{5} R\left(\omega^{s}-\omega_{0}^{s}\right) .
$$

This means that the after-impact velocities can be expressed by two numbers:

$$
e_{z}=-\frac{v_{z 1}^{s}}{v_{z 0}^{s}}, \quad e_{x}=-\frac{v_{x 1}^{s}+R \omega_{1}^{s}}{v_{x 0}^{s}+R \omega_{0}^{s}}=-\frac{v_{k 1}^{s}}{v_{k 0}^{s}} .
$$


For the normal contact problem the coefficient of restitution $e_{z}$ is used. In tangential direction the velocity of the contact point is the major variable. The ratio of the contact point's velocity after and before impact $e_{x}$ is the second number used in this paper. The tangential and angular velocity after the impact can be calculated from this number.

The viscoelastic half-space is modeled by the linear standard-model. The Maxwell-Element and the Kelvin-Voigt-Element are included as special cases. For the linear standard-model the time dependent relaxation modulus to shear has the following form:

$$
G(t)=G_{\infty}+G \exp \left(-\frac{t}{\tau}\right)
$$

It turns out that the numerical solution for $e_{x}$ and $e_{z}$ can be expressed by the following two dimensionless numbers:

$$
\delta_{1}=\eta\left(\frac{R v_{z 0}^{s}}{m^{2} G^{3}}\right)^{1 / 5}, \quad \delta_{2}=\eta\left(\frac{R v_{z 0}^{s}}{m^{2} G_{\infty}^{3}}\right)^{1 / 5}, \quad \eta=\tau G .
$$

At first glance it seems to be unreasonable that neither the tangential nor the angular velocity appears therein. But, under consideration that normal and tangential contact problems are decoupled, at least the coefficient of restitution $e_{z}$ cannot be dependent on any tangential velocity. Then again, the tangential problem is, under the assumption of a full stick regime, only dependent on the contact point's velocity and the contact area's shape during the impact. Since the system is linear, the magnitude of the contact point's velocity has no significance. It is finally used for normalization of $e_{x}$, as this has been done in the definition stated before.

Both numbers, $\delta_{1}$ and $\delta_{2}$, can be adapted to the special cases of the material model. The Maxwell-Element is obtained if $G_{\infty}$ tends towards zero, which means that $\delta_{2}$ becomes infinite. The Kelvin-Voigt-Element is obtained if $G$ tends toward infinity but $\eta$ is held constant. Since $\delta_{1} \sim \eta G^{-3 / 5}$ and $\delta_{2} \sim \eta$, the parameter $\delta_{1}$ becomes zero. Finally in the elastic case $\eta$ can be obtained to be either zero or infinity, which correspondents to an infinite soft or an infinite stiff damper. This means, expressed by the parameters, that either $\delta_{2}$ becomes zeros (and $\delta_{1}$ is finite, the elastic constant is $G_{\infty}$ ) or $\delta_{1}$ tends towards infinite (and $\delta_{2}$ is finite, the elastic constant is then equal to $\left.G_{\infty}+G\right)$. Summarized this means:

$$
\begin{array}{ll}
\text { Maxwell-Element: } & \delta_{2} \rightarrow \infty \\
\text { Kelvin-Voigt-Element: } & \delta_{1} \rightarrow 0 \\
\text { Elastic Spring: } & \delta_{1} \rightarrow \infty \text { or } \delta_{2} \rightarrow 0
\end{array}
$$

\section{THE BOUNDARY ELEMENT METHOD}

The used BEM is based on the fundamental solution for a point force acting on a viscoelastic, incompressible half-space [13-15]. Let $x$ and $y$ be Cartesian coordinates on the surface of a half-space. A point force with components $F_{x}, F_{y}$ and $F_{z}$, is applied at the centre of the mentioned coordinate system and causes the following deflection of the surface: 


$$
\left.\begin{array}{l}
4 \pi u_{x}=\frac{2}{r} \varphi_{x}+\left(\frac{x^{2}}{r^{3}}-\frac{1}{r}\right) \varphi_{x}+\frac{x y}{r^{3}} \varphi_{y}, \quad 4 \pi u_{z}=\frac{\varphi_{z}}{r}, \\
\varphi_{i}=F_{i} J(t), \quad r=\sqrt{x^{2}+y^{2}}
\end{array}\right\} .
$$

These equations can only be applied, if the acting force is added at time zero and the force is held to be constant. From this solution, the elastic fundamental solution can be obtained if $J(t) \rightarrow 1 / G$ is substituted. Herein $J(t)$ is the time dependent creep function for shear:

$$
J(t)=J_{0}+\frac{t}{\eta}+J\left(1-e^{-t / \lambda}\right) .
$$

This creep function (8) is related to the time dependent relaxation modulus $G(t)$ given in Eq. (4). In the case of the standard-material:

$$
J_{0}=\frac{1}{G+G_{\infty}}, \quad J=J_{0} \frac{G}{G_{\infty}}, \quad \lambda=\frac{\tau}{J_{0} G_{\infty}}, \quad \eta \rightarrow \infty,
$$

and in case of the Maxwell-element:

$$
J_{0}=\frac{1}{G}, \quad J=0, \quad \eta=\tau G .
$$

The fundamental solution for the viscoelastic material looks similar to the one from elastic material. Especially the dependency in space is the same. With this conclusion many schemes from elastic contact mechanics can be used. Firstly, a standard procedure is to integrate the fundamental solution (Boussinesq) over a rectangle, assuming constant pressure [16]. This solution (Love) can be superimposed to find the deflection for an arbitrary, but piecewise constant, pressure distribution [17]. To speed this up, it is common practice to interpret this procedure as a convolution, which can be carried out on massive parallel processing units [18-20]. The next, more complicated problem arising in the field of elastic contact mechanic is the opposite task: Solve the indentation problem of a rigid indenter pressed in an elastic half-space with an a priori unknown contact area. Then conjugate gradient algorithms are used, like the modified form by Polonsky and Keer [21]. The procedures named above are supposed to be known and are used in the following calculations.

The method described above can be applied to the viscoelastic case, if the acting forces are kept constant during one time step. The overall solution will be superimposed by adding the elastic solution multiplied with the time dependent creep function delayed by the time span since the force has been applied. At this point, a sum appears whose numerical costs grow linear in simulation time. By utilizing the special form of the creep function used here, an iterative algorithm can be derived. By applying this algorithm, only the previous time step is needed, and the contacting indenter shape is modified by a viscoelastic term. By using this method, the normal contact can be solved. A description and the application to the tangential sliding of an indenter can be found in [22]. The algorithm is shown below: 


$$
\left.\begin{array}{l}
u_{z, n}=\frac{J_{\infty}}{J_{0}} f_{n}+\underbrace{\frac{1}{J_{0} \eta} \sum_{i=0}^{n-1} \overbrace{\left(t_{i+1}-t_{i}\right)}^{h_{t}} f_{i}}_{a_{n}^{z}}-\underbrace{\frac{J}{J_{0}} \sum_{i=0}^{n}\left(f_{i}-f_{i-1}\right) \exp \left(-\frac{t_{n}-t_{i}}{\lambda}\right)}_{b_{n}^{z}} \\
u_{z, n+1}=\frac{J_{\infty}}{J_{0}} f_{n+1}+\underbrace{+\underbrace{z}_{n}+\frac{1}{J_{0} \eta} h_{t} f_{n}}_{a_{n+1}^{z}}-\underbrace{\exp \left(-\frac{h_{t}}{\lambda}\right) b_{n}^{z}+\left(f_{n+1}-f_{n}\right) \frac{J}{J_{0}}}_{D^{z}} \\
=f_{n+1}+\underbrace{a^{z}}_{a_{n+1}^{z}-\exp \left(-\frac{h_{t}}{\lambda}\right) b_{n}^{z}+f_{n} \frac{J}{J_{0}}}
\end{array}\right\} .
$$

Herein $u_{z, n}$ is the normal deflection of the surface and $f_{n}$ is the deflection due to a pressure distribution $p_{n}$ - each at time $t_{n}$. Furthermore, $a$ and $b$ are variables from the previous time step, initialized with value zero at the beginning of the simulation, and it is $J_{\infty}=J_{0}+J$. As can be seen in the last line of Eq. (11), deformation $D^{z}$ has to been taken into account to include viscoelastic behavior (in the elastic case $D^{z}$ is equal to zero). The only unknown term in Eq. (11) is $f_{n+1}$, which means that pressure distribution $p_{n+1}$ is unknown. This can be calculated with the elastic algorithms mentioned before. It should be noted that this algorithm can handle only materials with a finite modulus for instant deformation $(G(0)<\infty$ and $J(0)>0)$. That excludes the Kelvin-Element because it does not fit into the scope of this algorithm.

The tangential contact is very similar to the normal one. Only the calculation of the deflection in tangential direction $u_{x}$ has to be adopted. In all simulations carried out here, the deformation - perpendicular to the plane of the motion - is neglected. It has been shown for the case of parabolic bodies that this assumption causes only a negligible error [23,24].

At this point, the contact problem itself is solved. For the integration in time both an explicit Euler scheme and the velocity Verlet algorithm have been used. In comparison, they show no difference in the global error of the velocities at the end of the simulation and in the contact time itself. For an estimation of step size $h_{t}$ the solution of the elastic impact problem has been used:

$$
10^{4} h_{t}=T_{c} \approx T_{c, \text { elastic }}=2.87\left(\frac{m^{2}}{R(4 G(t))^{2} v_{z 0}^{s}}\right)^{1 / 5} .
$$

Since the shear modulus is time dependent, one has to start with $G(0)$ and iteratively set $t \rightarrow T_{c}$. With the elastic solution also an approximation for contact radius $a$ can be found:

$$
a \approx a_{\text {elastic }}=\sqrt{R \delta}, \quad \delta=\frac{T_{c, \text { elastic }} v_{z 0}^{s}}{2.94} .
$$

The geometric discretisation has been chosen in a way that $256 \times 256$ points are used. A comparison with a finer discretisation shows only a slight improvement of the error. For implementation it has to be considered that the total deflection in normal direction within the contact area is known at every time step since the indentation depth of the sphere is known. Contrariwise in tangential direction: the points coming into contact have a pre-deformation through coupling to the points within the contact area from the previous time steps. This can be handled by adding only the current increment of tangential movement at the boundary of the sphere in each time step. 


\section{RESULTS}

The simulation carried out shows that the assumptions, condensed in formula (3) and (5), are correct. This has been proven by choosing random simulation parameters, that all coincide in the same functional relation. This relation has been fitted to the numerical data with two functions, one for each number $e_{x}$ and $e_{z}$. In the following contour plots (Figs. 2 and 3 ) the obtained data and the fitting are shown. The fitting function and the parameter are given below.

The fitting function for the coefficient of restitution in normal direction $e_{z}$ is (parameters are given in Table 1):

$$
\left.\begin{array}{l}
e_{z} \approx \frac{1}{2} \tanh \left(c_{1} \rho+c_{2}\right)+\frac{1}{2} \\
\rho=\sqrt{x^{2}+y^{2}} \\
x=\max \left\{0, \log _{10}\left(\delta_{1}\right)-\delta_{1}^{p}\right\} \\
y=\max \left\{0, \frac{\log _{10}\left(\delta_{2}\right)}{\sqrt{2}}-\delta_{2}^{p}\right\}
\end{array}\right\}
$$
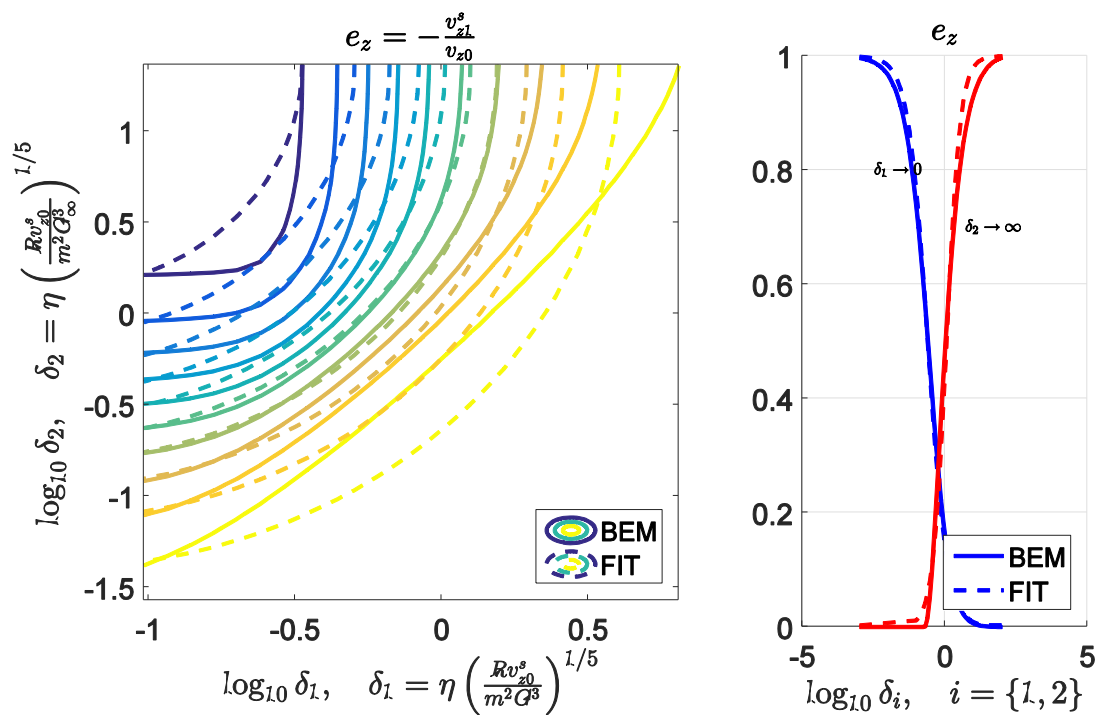

Fig. 2 Contour lines of the coefficient of restitution $e_{z}$ (left hand side). Special case of the Kelvin-Voigt-Element $\left(\delta_{1} \rightarrow 0\right)$ and the Maxwell-Element $\left(\delta_{2} \rightarrow \infty\right)$ (right hand side)

Table 1 List of the fitting parameters for the coefficient of restitution in normal direction $e_{z}$ corresponding to the fitting function given in (14)

\begin{tabular}{lcccc}
\hline Parameter & $\delta_{1}{ }^{p}$ & $\delta_{2}{ }^{p}$ & $c_{1}$ & $c_{2}$ \\
\hline Value & -1.342 & 0.9583 & 2.079 & -2.892 \\
\hline
\end{tabular}


The fitting function for the coefficient of restitution in tangential direction $e_{x}$ is (parameters are given in Table 2):

$$
\begin{aligned}
& e_{x} \approx e_{x}^{e l}+\left(e_{x}^{\min }-e_{x}^{e l}\right) e_{x}^{\alpha}+\left(e_{x}^{\max }-e_{x}^{e l}\right) e_{x}^{\beta}, \\
& e_{x}^{\alpha}=\frac{c_{1}}{2}\left[1+\tanh \left(c_{3}+c_{4} y\right)\right] \exp \left[-\left(\frac{x}{c_{2}}\right)^{2}\right], \\
& \left.e_{x}^{\beta}=\frac{1}{4}\left\{1+\tanh \left(c_{5}-c_{6} y\right)+2 \exp \left[-\left(\frac{y}{c_{7}}\right)^{2}\right]-2\right\}\right\} \\
& \quad \times\left\{1+\tanh \left(c_{8}-c_{9} x\right)\right\}, \\
& x=\log _{10}\left(\delta_{1}\right)-\delta_{1}^{p} \\
& y=\log _{10}\left(\delta_{2}\right)-\delta_{2}^{p}
\end{aligned}
$$
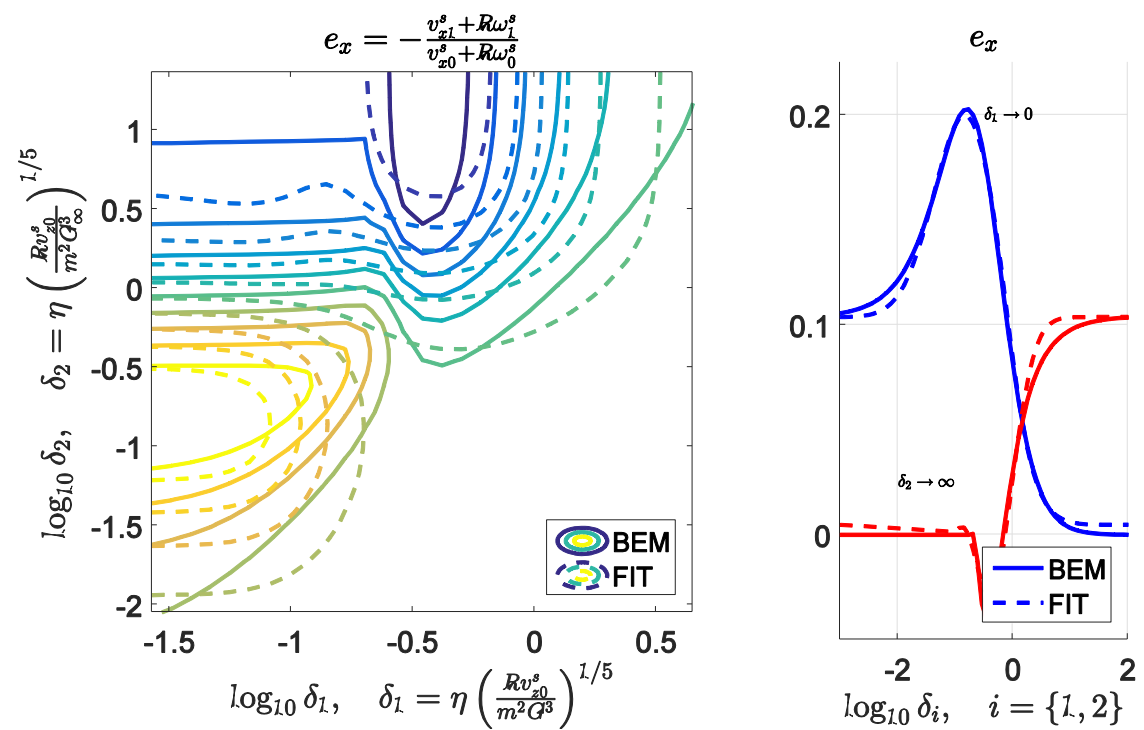

Fig. 3 Contour lines of the coefficient of restitution $e_{x}$ (left hand side). Special case of the Kelvin-Voigt-Element $\left(\delta_{1} \rightarrow 0\right)$ and the Maxwell-Element $\left(\delta_{2} \rightarrow \infty\right)$ (right hand side)

Table 2 List of the fitting parameters for the coefficient of restitution in normal direction $e_{x}$ corresponding to the fitting function given in Eq. (15)

\begin{tabular}{lccccc}
\hline Parameter & $e_{x}^{\min }$ & $e_{x}^{e l}$ & $e_{x}^{\max }$ & $\delta_{1}{ }^{p}$ & $\delta_{2}{ }^{p}$ \\
\hline Value & -0.0399 & 0.1038 & 0.2028 & -0.4 & -0.8 \\
\hline Parameter & $c_{1}$ & $c_{2}$ & $c_{3}$ & $c_{4}$ & $c_{5}$ \\
\hline Value & 2.335 & 0.5406 & -2.29 & 2.253 & 1.718 \\
\hline Parameter & $c_{6}$ & $c_{7}$ & $c_{8}$ & $c_{9}$ & \\
Value & 2.335 & 0.7999 & -2.105 & 4.000 \\
\hline
\end{tabular}




\section{CONCLUSIONS}

The BEM has been extended to the normal and tangential contact problem of viscoelastic media. The approach has different advantages: It implements a relatively simple adaptation to the existing BEM procedures developed for elastic media and its applicability to any other transient problem dealing with time dependent viscoelastic behavior modeled by the Prony series. Exemplary the impact of a sphere with an elastomer, modeled by a Maxwell-element and a standard-model, has been investigated. The obtained compact and elegant solution is given in form of a fitting function for further use.

\section{REFERENCES}

1. Hertz, H., 1882, Über die Berührung fester elastischer Körper, Journal für die reine und angewandte Mathematik, 92, pp. 156-171.

2. Deresiewicz, H., 1968, A Note on Hertz's Theory of Impact, Acta Mechanica, 6, pp. 110-112.

3. Mindlin, R.D., 1949, Compliance of Elastic Bodies in Contact, Journal of Applied Mechanics, 16, pp. 259-268.

4. Maw, N., Barber, J.R., Fawcett, J.N., 1976, The Oblique Impact of Elastic Spheres, Wear, 38(1), pp. 101-114.

5. Maw, N., Barber, J.R., Fawcett, J.N., 1977, The Rebound of Elastic Bodies in Oblique Impact, Mechanical Research Communications, 4(1), pp. 17-22.

6. Maw, N., Barber, J.R., Fawcett, J.N., 1981, The Role of Elastic Tangential Compliance in Oblique Impact, Journal of Lubrication Technology, 103(1), pp. 74-80.

7. Jäger, J., 1994, Analytical Solutions of Contact Impact Problems, Applied Mechanics Review, 47(2), pp. 35-54

8. Lyashenko, I.A., Popov, V.L., 2015, Impact of an elastic sphere with an elastic half space revisited: Numerical analysis based on the method of dimensionality reduction, Sci. Rep., 5, 8479.

9. Willert, E., Popov, V.L., 2016, Impact of an elastic sphere with an elastic half space with a constant coefficient of friction: Numerical analysis based on the method of dimensionality reduction, Journal of Applied Mathematics and Mechanics (ZAMM), 96(9), pp. 1089-1095.

10. Hunter, S. C., 1960, The Hertz problem for a rigid spherical indenter and a viscoelastic half-space, Journal of the Mechanics and Physics of Solids, 8(4), pp. 219-234.

11. Sabin, G. C. W., 1987, The impact of a rigid axisymmetric indenter on a viscoelastic half-space, International Journal of Engineering Science, 25(2), pp. 235-251.

12. Calvit, H. H., 1967, Numerical solution of the problem of impact of a rigid sphere onto a linear viscoelastic half-space and comparison with experiment, International Journal of Solids and Structures, 3(6), pp. 951-960.

13. Gasanova, L., Gasanova, P., Talybly, L., 2011, Solution of a viscoelastic boundary-value problem on the action of a concentrated force in an infinite plane, Mechanics of Solids, 46(5), pp. 772-778.

14. Peng, Y., Zhou, D., 2012, Stress Distributions Due to a Concentrated Force on Viscoelastic Half-Space, Journal of Computation \& Modeling, 2(4), pp. 51-74.

15. Talybly, L., 2010, Boussinesq's viscoelastic problem on normal concentrated force on a half-space surface, Mechanics of Time-Dependent Materials, 14(3), pp. 253-259.

16. Johnson, K. L., 1985, Contact Mechanics, Cambridge University Press, Cambridge.

17. Pohrt, R., Li, Q., 2014, Complete Boundary Element Formulation for Normal and Tangential Contact Problems, Physical Mesomechanics, 17(4), pp. 334-340.

18. Cho, Y. J., Koo, Y. P., Kim, T. W., 2000, A new FFT technique for the analysis of contact pressure and subsurface stress in a semi-infinite solid, KSME International Journal, 14(3), pp. 331-337.

19. Liu, S.,Wang, Q., Liu, G., 2000, A versatile method of discrete convolution and FFT DC-FFT for contact analyses, Wear, 243(1-2), pp. 101-111.

20. Wang, W.Z., Wang, H., Liu, Y.C., Hu, Y.Z., Zhu, D., 2003, A comparative study of the methods for calculation of surface elastic deformation, Proceedings of the Institution of Mechanical Engineers, Part J: Journal of Engineering Tribology, 217, 145-154

21. Polonsky, I., Keer, L., 1999, A numerical method for solving rough contact problems based on the multi-level multi-summation and conjugate gradient techniques, Wear, 231(2), 206-219.

22. Kusche, S., 2016, Frictional force between a rotationally symmetric indenter and a viscoelastic half-space. ZAMM - Journal of Applied Mathematics and Mechanics, pp. 1-14.

23. Johnson, K.L., 1955, Surface interaction between elastically loaded bodies under tangential forces, Proc. R. Soc. A., 230, 531-548.

24. Munisamy, R.L., Hills, D.A., Nowell, D., 1994, Static axisymmetric Hertzian contacts subject to shearing forces, ASME J. Appl. Mech., 61(2), pp. 278-283. 